\title{
Towards a target label-free suboptimum oligonucleotide displacement-based detection system
}

\author{
Mònica Mir • Pablo Lozano-Sánchez • Ioanis Katakis
}

Received: 29 February 2008 /Revised: 28 March 2008 / Accepted: 3 April 2008 / Published online: 3 May 2008

(C) Springer-Verlag 2008

\begin{abstract}
A novel method for the future development of label-free DNA sensors is proposed here. The approach is based on the displacement of a labelled suboptimum mutated oligonucleotide hybridised with the immobilised biotincapture probe. The target fully complementary to the biotin-capture probe can displace the labelled oligonucleotide causing a subsequent decrease of the signal that verifies the presence of the target. The decrease of signal was demonstrated to be proportional to the target concentration. A study of the hybridisation of mutated and complementary labelled oligonucleotides with an immobilised biotin-capture probe was carried out. Different kinetic and thermodynamic behaviour was observed for heterogeneous hybridisation of biotin-capture probe with complementary or suboptimum oligonucleotides. The displacement method evaluated colourimetrically achieved the objective of decreasing the response time from $1 \mathrm{~h}$ for direct hybridisation of 19-mer oligonucleotides in the direct enzyme-linked oligonucleotide assay (ELONA) to $5 \mathrm{~min}$ in the case of displacement detection in the micromolar concentration range.
\end{abstract}

Keywords Biosensor platform · Displacement · DNA . Label-free target $\cdot$ ELONA $\cdot$ Colourimetric detection

M. Mir · P. Lozano-Sánchez $\cdot$ I. Katakis

Bioengineering and Bioelectrochemistry Group,

Departament d'Enginyeria Química, Escola Tècnica Superior

d'Enginyeria Química, Universitat Rovira i Virgili,

Avd. Països Catalans, 26,

43007 Tarragona, Spain

M. Mir $(\bowtie)$

Max Planck Institute for Polymer Research,

Ackermannweg 10,

55128 Mainz, Germany

e-mail: mir@mpip-mainz.mpg.de

\section{Introduction}

DNA biosensors are the subject of intense research and development, which has already produced several commercial platforms. Possible applications of these biosensors range from diagnosis of genetic diseases [1], detection of infectious agents [2], study of genetic predisposition [3], development of a personalised medicine, detection of differential genetic expression, forensic science [4], drug screening [5], food safety [6], or even environmental monitoring [7].

Most DNA sensor platforms require previous labelling of the DNA target. This step requires not only some molecular biology knowledge but costly laboratory equipment; hence efforts towards the design of label-free DNA detection systems to be integrated in cheaper, more accessible biosensor platforms are relevant.

Some examples of general label-free detection are reported in the literature. The majority of them are related to surface plasmon resonance and quartz crystal microbalance transducers [8-11]. These approaches allow the detection of any biomolecule interaction without the need to previously label the target; however, these transducers are costly and do not appear suitable for implementation in portable or light equipment. Colourimetric transducers are cheaper alternative that moreover allow the detection of an oligonucleotide target without labelling. A sandwich approach attempted through a lateral-flow assay has been reported [12]. In this system three DNA strands are involved. One of the DNA strands that hybridise with the target was attached to liposomes encapsulating a reporter molecule, the other strand linking with the target was immobilised on the surface. When the target is present the strand with the liposomes is immobilised on the surface and yields a visible magenta band at the capture zone. The use of intercalators (redox molecules that can be entrapped selectively in the 
double strand of DNA molecules, revealing the hybridisation of the unlabelled target) has also been reported using a colourimetric transduction technique. Hoechst 33258 [13], daunomycin [14] and methylene blue [15] are some examples of intercalators used to detect DNA hybridisation in biosensors. The use of colloidal gold nanoparticles has also been applied in a label-free colourimetric method to detect DNA hybridisation [16]. ssDNA stabilises colloidal suspensions and addition of salt does not produce an aggregation of the colloid. However, dsDNA adsorbed on colloidal gold is not stable. When salt is added a change of colour from red to blue is observed, indicating the aggregation of colloids. The hybridisation of the target ssDNA to dsDNA can thus be detected. However in both sandwich and intercalation strategies an incubation step after the target hybridisation is required, making this technique more time consuming.

Another alternative is fluorescent molecular beacons, which provide a label-free platform and real-time detection of the hybridisation. Molecular beacons are single-stranded oligonucleotide probes that possess a stem-loop structure. The loop portion is complementary to ssDNA target, while the stem is formed by five to seven base pairs from two complementary arm sequences that are in the two ends of the loop. A fluorophore molecule is attached to the end of the strand, while a quencher molecule is attached to the opposite end. The stem maintains close proximity of the two moieties, causing fluorescence to be quenched by energy transfer. When the complementary target hybridises with the beacon probe, the stem is opened leading to restoration of fluorescence. Fluorescent molecular beacons have been used in single biosensors to monitor the reaction dynamics of the DNA hybridisation process [17], as well as in arrays for detection of multiple analytes [18]. However reproducibility problems and background noise due to incomplete quenching of the fluorescing component have also been reported $[17,19]$.

A promising novel methodology to develop label-free DNA detection systems is reported here. This system is based on the concept of displacement. Displacement methodology has long been used in enzyme-linked immunoassays (ELISA) [20]. In these assays a biocapture molecule is immobilised on polystyrene or streptavidin-modified plates. The enzyme-linked target is incubated with the immobilised capture probe and interacts specifically with it. The noninteracting molecules are washed and the interaction is detected colourimetrically after the addition of the enzyme substrate.

However, displacement assays for oligonucleotide detection have been very underexploited. Friz Biochem commercialised the EDDA $^{\mathrm{TM}}$ (electrically detected displacement assay) for single nucleotide polymorphism (SNP) detection. The sensor was based on displacement of the target hybridised with the capture probe. The SNP that contains the target produces instability in the duplex with the capture probe, which allows the displacement of the target by a labelled oligonucleotide complementary to the capture probe [21].

In this work a labelled mutated oligonucleotide is hybridised with the immobilised capture probe. The complementary target has higher affinity for the capture probe, so it can displace the labelled oligonucleotide. Thus the decrease in the signal can be associated with a proportional increase in the target concentration. These displacement strategies do not require a step after the addition of the target, which makes them reagentless and easier to use. A proof of principle approach to the exploitation of the displacement methodology in oligonucleotide label-free detection is reported here. The utilisation of relatively short oligonucleotides (19-mers) has been used to illustrate how the hybridisation-dehybridisation conditions can dictate the success of the displacement methodology. A study of the hybridisation of the capture probe with mutated and complementary oligonucleotides was also carried out to optimise the system.

\section{Experimental}

\section{Materials}

\section{Reagents}

The following oligonucleotide sequences used in the displacement assay for the Mycobacterium tuberculosis gene region that confers resistance to rifampicin were ordered from Eurogentec (Belgium) and purified by HPLC:

1. 5'-Biotin-AGCCAGCTGAGCCAATTCA-3' (biotincapture probe - a 19-mer biotin-labelled capture probe oligonucleotide.

2. 5'-TGAATTGGCTCAGCTGGCT-3' (complementary target) - a 19-mer complementary oligonucleotide.

3. 5'-Thiol- $\mathrm{C}_{6}$-TGAATTGGCTCAGCTGGCT-3'-a 19mer thiol-labelled complementary oligonucleotide that was subsequently bioconjugated with maleimide-activated horseradish peroxidase (maleimide-HRP) leading to an HRP-labelled oligonucleotide termed complementaryHRP.

4. 5'- Thiol- $\mathrm{C}_{6}$-TGAGTGGGCTCGGGTGGCT-3'-a 19-mer thiol-labelled mutated oligonucleotide that was also subsequently bioconjugated with maleimide-HRP leading to an HRP-labelled oligonucleotide termed mutated-HRP. This sequence has four mutations with respect to the totally complementary sequence.

Sodium phosphate buffer (PBS), sodium chloride $(\mathrm{NaCl})$, Denhard's solution, maleimide-HRP, 3,3',5,5'-tetramethylbenzidine liquid substrate (TMB), tris(hydroxymethyl)ami- 
nomethane hydrochloride (Tris-HCl), polyethylene glycol sorbitan monolaurate (Tween), ethylenediaminetetraacetic acid (EDTA), saline sodium citrate buffer (SSC) and milk powder were purchased from Sigma. The Sephadex G25 DNA grade resin (17-0853-01) was purchased from Amersham Pharmacia Biotech. Streptavidin-coated plates were supplied by Labsystems.

\section{Equipment}

The oligonucleotide bioconjugation with HRP was monitored with an 8453 spectrophotometer (Hewlett Packard) with UV-Visible system HP845x software. Colourimetric detection of HRP-labelled DNA hybridisation by ELONA was performed at $405 \mathrm{~nm}$ with an ELISA plate reader SpectraMax 340PC from Molecular Devices running SoftMax Pro software.

\section{Methods}

\section{Oligonucleotide-HRP bioconjugation}

The complementary and mutated oligonucleotides were purchased with a 5'-thiol and a six-carbon-atom spacer. These were labelled with HRP using maleimide activation [22]. The maleimide-activated peroxidase was dissolved in $100 \mathrm{mM}$ sodium phosphate buffer (PBS), $30 \mathrm{mM}$ EDTA and $10 \mathrm{mM} \mathrm{NaCl}$ at $\mathrm{pH} 6.5$ and mixed with a ten-fold molar excess of thiol-labelled oligonucleotide solution with respect to maleimide-HRP. The maleimide-activated peroxidase reacts with the thiol groups to covalently attach the HRP to the oligonucleotide. The solution was left to stand for $12 \mathrm{~h}$ in darkness and was then purified with a Sephadex G25 gel filtration column. The concentration of the resulting HRP-labelled oligonucleotide and the activity of enzyme labelled was determined spectrophotometrically at $403 \mathrm{~nm}$ and $260 \mathrm{~nm}$, respectively.

\section{Immobilisation of capture probe and mutated-HRP}

Based on ELISA, ELONA uses a similar method but to detect oligonucleotides. Streptavidin plates were used to immobilise the biotin-labelled capture probe. A $50 \mu \mathrm{L}$ volume of $0.2 \mu \mathrm{g} \mathrm{mL}^{-1}$ biotin-capture probe solution was left to bind for $15 \mathrm{~min}$ at $25{ }^{\circ} \mathrm{C}$. A $50 \mu \mathrm{L}$ aliquot of $10 \mu \mathrm{g} \mathrm{mL}^{-1}$ mutated-HRP oligonucleotide in $10 \mathrm{mM}$ Tris$\mathrm{HCl}, 1 \mathrm{mM}$ EDTA, $0.3 \times \mathrm{SSC}$ and $2 \times$ Denhardt's solution at $\mathrm{pH} 7.5$ (hybridisation buffer) was added and left to incubate for $1 \mathrm{~h}$ at $50{ }^{\circ} \mathrm{C}$. Hybridisation of mutated-HRP and complementary-HRP was optimised for various concentrations, times and temperatures to obtain the best conditions for prehybridisation of mutated-HRP and its subsequent displacement. The prehybridisation of mutat-
ed-HRP was detected with the addition of $100 \mu \mathrm{L}$ of TMB incubated for $30 \mathrm{~min}$ at room temperature. The coloured reagent generated from the reaction of the TMB with HRP was detected spectrophotometrically at $405 \mathrm{~nm}$. Afterwards $50 \mu \mathrm{L}$ of $30 \mu \mathrm{g} \mathrm{mL}^{-1}$ complementary target in hybridisation buffer was incubated for different times and at varying temperatures to optimise the displacement of the mutated-HRP, followed by the detection with TMB in the same manner. A washing step with $100 \mathrm{mM}$ Tris- $\mathrm{HCl}$ $\mathrm{pH} 7.5,150 \mathrm{mM} \mathrm{NaCl}$ and $1 \%$ Tween (wash buffer) was performed between all incubation steps to wash out all the unreacted molecules.

\section{Results and discussion}

The method of detection by displacement requires the prehybridisation of the biotin-capture probe immobilised on the surface with a mutated-HRP oligonucleotide. Owing to the higher affinity of the target that is fully complementary to the biotin-capture probe, the suboptimum label can be displaced when the complementary target is introduced into the system (see Fig. 1). The decrease of the signal should verify the presence of the target and should be proportional to its concentration.

In order to obtain a functional displacement system it is important to have a prehybridisation of the mutated-HRP that is stable enough to maintain the duplex until the target can come into contact with the biotin-capture probe. This prevents the displacement of mutated-HRP by buffer or other noncomplementary oligonucleotides. At the same time, this duplex should be suitably displaceable by the target that is fully complementary to the biotin-capture probe.

\section{Suboptimum mutated sequence choice}

Affinity drives the duplex formation between two oligonucleotide strands and depends on the number of mutations and the type and position of the mismatched base pair [23]. In a mutated duplex, the presence of mismatched base pairs affects the dissociation kinetic constant $\left(k_{\text {diss }}\right)$ more than the association constant $\left(k_{\text {ass }}\right)$ [24].

Santa Lucia and coworkers [25-27] reported the stability conferred by base pairs obtained from statistical simulation, according to the following sequence: $\mathrm{G}-\mathrm{C}>\mathrm{A}-\mathrm{T}>\mathrm{G}-\mathrm{G}>$ $\mathrm{G}-\mathrm{T}=\mathrm{G}-\mathrm{A}>\mathrm{T}-\mathrm{T}=\mathrm{A}-\mathrm{A}>\mathrm{T}-\mathrm{C}>\mathrm{A}-\mathrm{C}>\mathrm{C}-\mathrm{C}$. These results were experimentally confirmed by Tawa and Knoll [28], who obtained more stable duplexes from a $\mathrm{T}-\mathrm{G}$ mismatch than from a $\mathrm{T}-\mathrm{C}$ pair. $\mathrm{G}-\mathrm{A}, \mathrm{G}-\mathrm{G}$ and $\mathrm{G}-\mathrm{T}$ were demonstrated to be less destabilising mismatches than $\mathrm{C}$ $\mathrm{C}$ and $\mathrm{C}-\mathrm{T}$ [23], and $\mathrm{G}-\mathrm{A}$ and $\mathrm{G}-\mathrm{T}$ mismatches were detected to have better stability than $\mathrm{A}-\mathrm{A}, \mathrm{T}-\mathrm{T}, \mathrm{C}-\mathrm{T}$ and C-A [24]. In contrast with Santa Lucia and coworkers' 
Fig. 1 Schematic diagram of suboptimum HRP-labelled mutated oligonucleotide displaced by the complementary target

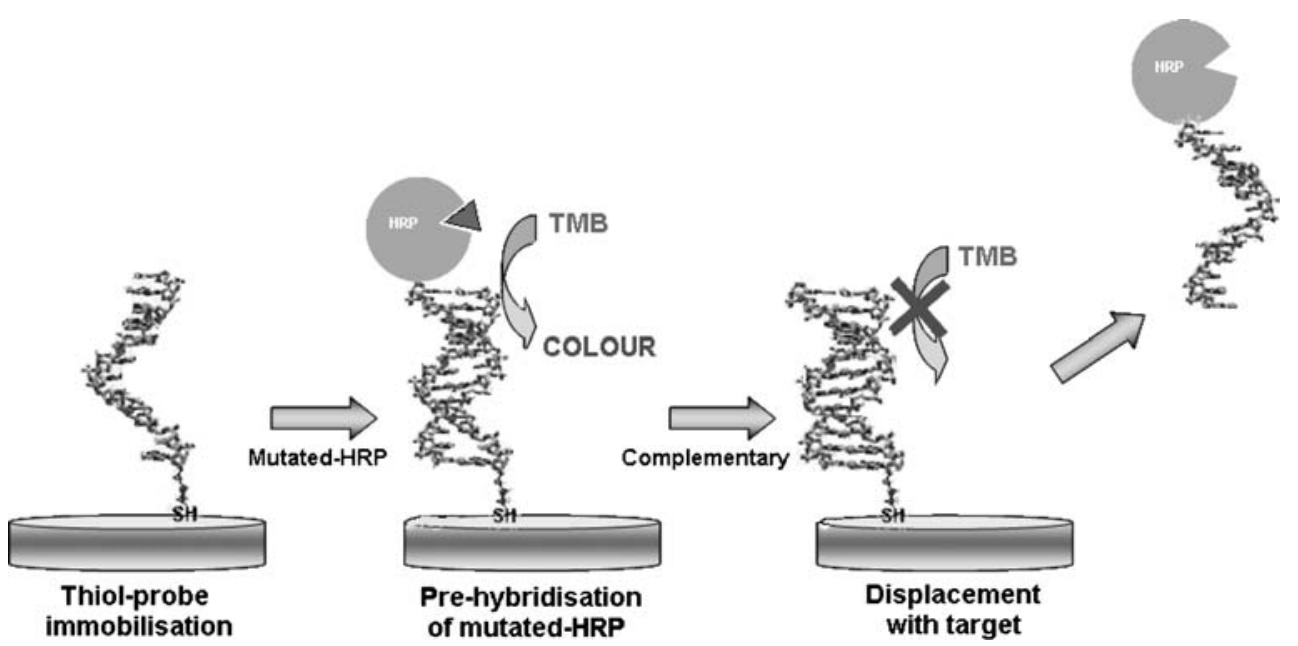

closer to the centre of the sequence, in both cases having two groups of five matches together in the 19-mer sequence, which can stabilise the duplex. However, the presence of mismatches to the $3^{\prime}$ end of the capture probe can promote the "unzipping" of the duplex.

Hybridisation of mutated and complementary HRP-labelled oligonucleotides with capture probe

Before carrying out displacement assays the conditions for hybridisation of complementary and mutated oligonucleotides were fine-tuned. Blocking agent, temperature and time of the hybridisation as well as concentration of oligonucleotides were optimised. Preliminary spectrophotometrical experiments were done to obtain a rough indication of the affinity of the biotin-capture probe with both oligonucleotides and establish the initial conditions of the ELONA experiments. Control experiments without biotin-capture probe and with and without blocking verified the same low nonspecific adsorption of labelled oligonucleotide HRP on the surface. Subsequently experiments were done without blocking in order to reduce steps and increase sensitivity.

High thermodynamic stability of the mutated-HRP duplex with the biotin-capture probe was observed. The response from the mutated-HRP-biotin-capture probe duplex is practically equal to the response from the complementary-HRP-biotin-capture probe duplex under the experimental conditions comprising a long hybridisation time $(1 \mathrm{~h})$ and low temperature $\left(25^{\circ} \mathrm{C}\right)$. This behaviour shows that the first goal of the probe design has been achieved i.e. a strong duplex with the suboptimum sequence; however, the question remains if the labelled oligonucleotide could be displaced. This behaviour could also suggest that hybridisation on solid supports is more complex than in solution [30,31].

The effect of temperature on the hybridisation of both strands (mutated-HRP and complementary-HRP) showed similar response between 15 and $35^{\circ} \mathrm{C}$ : a more efficient 
response (ca. 20-28\%) was obtained at higher temperatures from the complementary-HRP signal than the mutated-HRP signal.

These results could be explained by taking into account the melting temperature $\left(T_{\mathrm{m}}\right)$ values of both duplexes. $T_{\mathrm{m}}$ values obtained for the complementary-HRP sequence were $57{ }^{\circ} \mathrm{C}$ from the Howley equation [32] and $54{ }^{\circ} \mathrm{C}$ from the neighbouring bases relationship [33]. A reduction of $1{ }^{\circ} \mathrm{C}$ in the $T_{\mathrm{m}}$ for every $1 \%$ of base pair mutations in dsDNA has been reported [34]. Accordingly, the $T_{\mathrm{m}}$ of the mutated-HRP sequence might be around $36{ }^{\circ} \mathrm{C}$. Therefore at $50{ }^{\circ} \mathrm{C}$, the complementary-HRP is close to its $T_{\mathrm{m}}$, while the mutatedHRP should not hybridise so much at $14{ }^{\circ} \mathrm{C}$ above its $T_{\mathrm{m}}$. However, the $T_{\mathrm{m}}$ values and the assumption of the percentage of temperature decrease with mismatches were calculated for hybridisation duplexes in solution. Some examples in the literature demonstrate that the theory applicable to DNA hybridisation in solution cannot be applied for heterogeneous hybridisation. It was demonstrated that the classical equation of melting curve analysis could not be applied in the case of hybridisation of target with an immobilised capture probe on solid support, because it is valid only for equal concentrations of both strands [35]. Furthermore, the literature for solid-phase DNA oligonucleotide duplex formations suggests that the prenucleation state is greatly affected by temperature and an increase of temperature in all cases resulted in easier and faster hybridisation [36]. The different relationships between the mutations and the decrease of $T_{\mathrm{m}}$ with duplexes immobilised on a solid phase were studied [29]. $T_{\mathrm{m}}$ of DNA with adjacent mismatches showed a decrease of $1{ }^{\circ} \mathrm{C}$ in the $T_{\mathrm{m}}$ for every $0.5 \%$ mutations of base pairs in double-stranded DNA. However as mentioned before all mismatches cannot be treated in the same way, mainly due to the dependence on the type of mismatch and its position on the kinetic and thermodynamic stability of the DNA duplex.

The results of mutated-HRP and complementary-HRP hybridised with the biotin-capture probe at different temperatures suggest that a critical point is reached at temperatures higher than $35^{\circ} \mathrm{C}$ because either the thermodynamics or the nucleation of zippering kinetics are affected. As shown in Fig. 2 at higher temperatures the signal difference between mutated-HRP and the complementary-HRP increases with time. In addition, the difference of the signal is higher at longer times (see Fig. 3). It appears therefore that the hybridisation of the complementary-HRP is favoured at higher temperatures and longer incubation times.

These results agree with the literature $[37,38]$ in that hybridisation kinetics are significantly different for specific and nonspecific binding of labelled DNA to surface-bound oligonucleotides. It is reported that specific binding takes longer to reach hybridisation equilibrium than for the mismatched oligonucleotide. Increasing hybridisation time

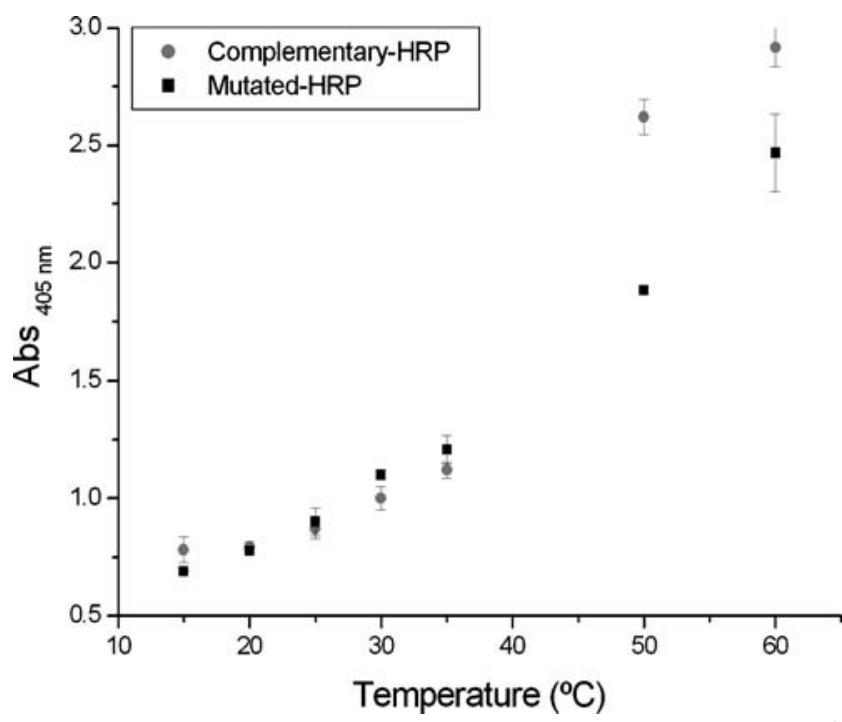

Fig. 2 Absorbance resulting from the hybridisation of $30 \mu \mathrm{g} \mathrm{mL} L^{-1}$ mutated-HRP and $30 \mu \mathrm{g} \mathrm{mL}^{-1}$ complementary-HRP with immobilised capture probe for $60 \mathrm{~min}$. Colourimetric detection was performed in all cases after $30 \mathrm{~min}$ incubation with $\mathrm{TMB}$ at room temperature at $405 \mathrm{~nm}(n=3)$

generally increases the specificity of the hybridisation. This behaviour could be partially explained with the model of oligonucleotide hybridisation on a solid support reported by Chan and coworkers [39]. Capture of the perfectly complementary target is modelled as a combined reaction-diffusion process. In this model there are two different mechanisms by which targets can hybridise with the complementary probes: the direct hybridisation from the solution and the

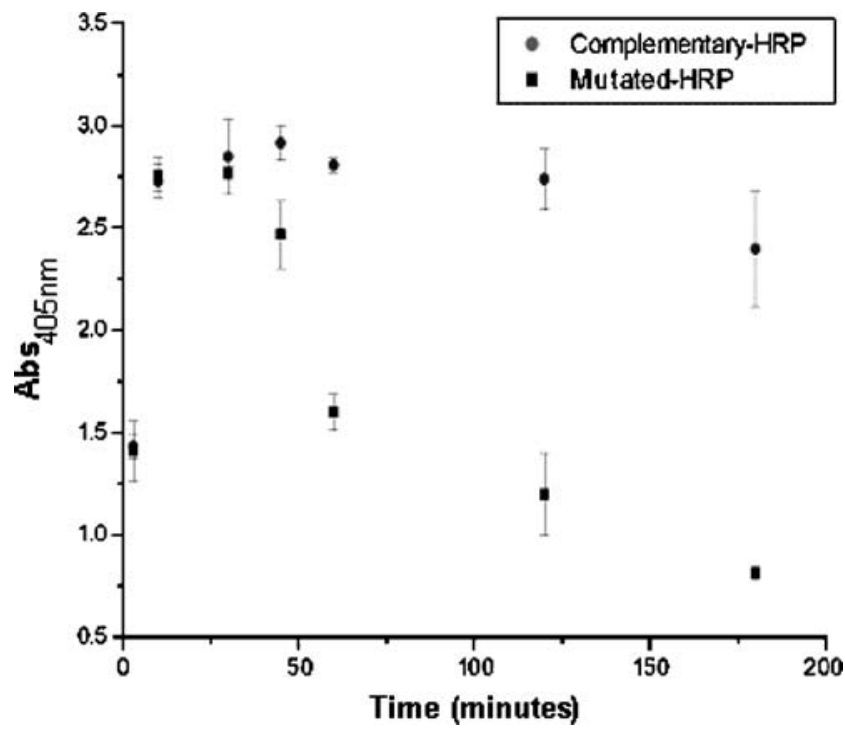

Fig. 3 Absorbance resulting from the extended interaction of $30 \mu \mathrm{g} \mathrm{mL}{ }^{-1}$ mutated-HRP and $30 \mu \mathrm{g} \mathrm{mL}^{-1}$ complementary-HRP with immobilised capture probe. Incubation time at $60{ }^{\circ} \mathrm{C}$. Colourimetric detection was performed after 30 min incubation with TMB at room temperature and at $405 \mathrm{~nm}(n=3)$ 


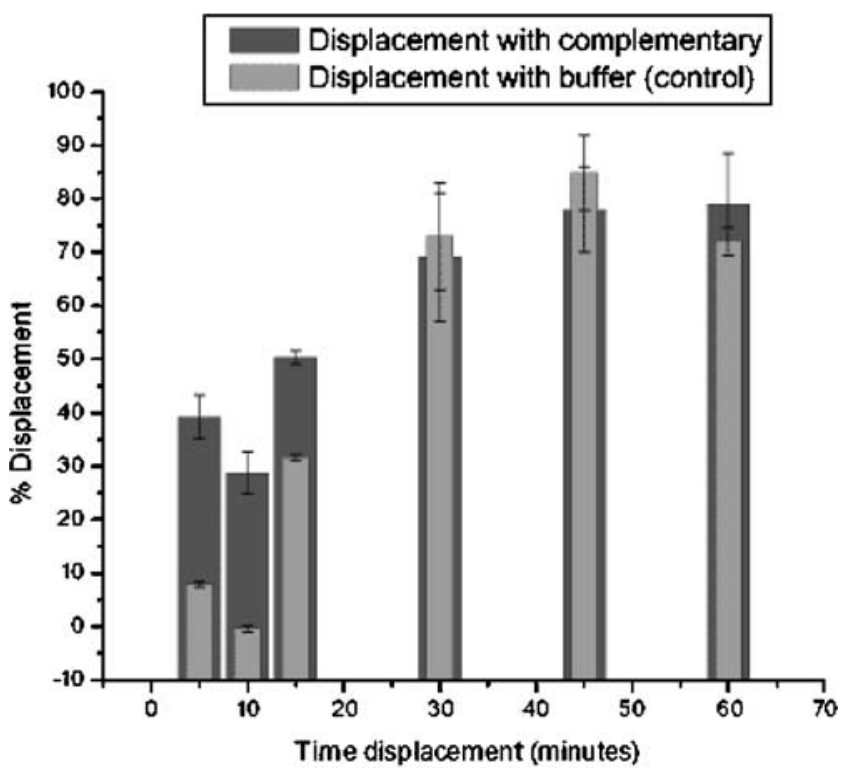

Fig. 4 Absorbance resulting from the displacement of prehybridised mutated-HRP with complementary target or buffer solution (control) as a function of time. Prehybridisation conditions $10 \mathrm{mg} \mathrm{mL} \mathrm{m}^{-1}$ mutated-HRP for $1 \mathrm{~h}$ at $50{ }^{\circ} \mathrm{C}$. Displacement conditions $30 \mu \mathrm{g} \mathrm{mL}{ }^{-1}$ of complementary target at $60{ }^{\circ} \mathrm{C}$ for various incubation times. Colourimetric detection was performed after $30 \mathrm{~min}$ incubation with $\mathrm{TMB}$ at room temperature and $405 \mathrm{~nm}(n=3)$

hybridisation by molecules that adsorb nonspecifically and then surface diffuse to the probe. Bloomfield et al. reported a rate constant for oligonucleotides association in solution of three orders of magnitude smaller than the diffusion-limited

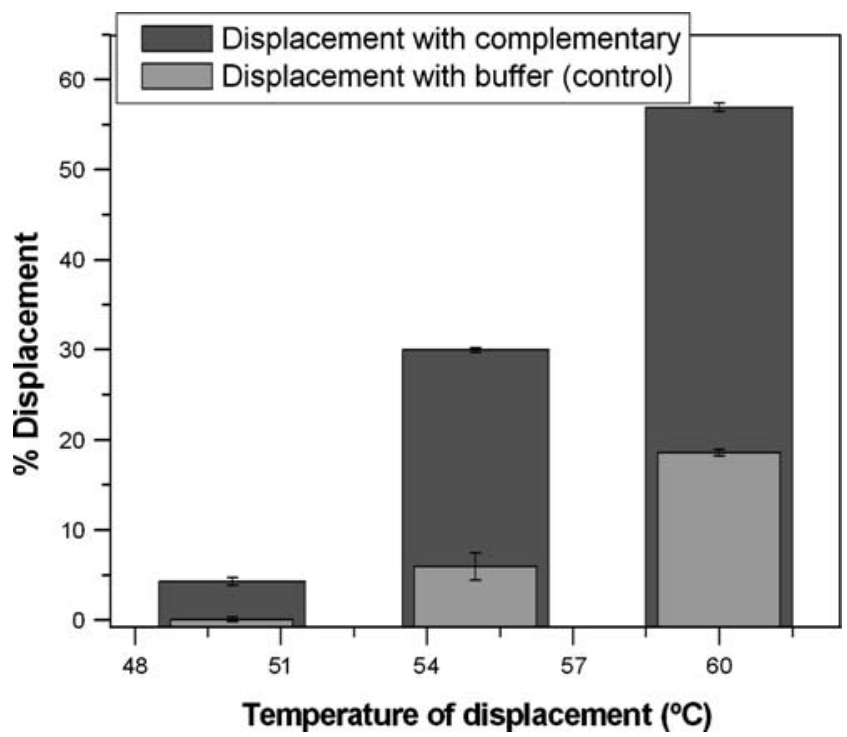

Fig. 5 Absorbance resulting from the displacement of prehybridised mutated-HRP with complementary target or buffer (control) as a function of the temperature. Prehybridisation conditions $10 \mathrm{mg} \mathrm{mL}^{-1}$ mutated-HRP for $1 \mathrm{~h}$ at $50{ }^{\circ} \mathrm{C}$. Displacement conditions $30 \mu \mathrm{g} \mathrm{mL}{ }^{-1}$ of complementary target for $5 \mathrm{~min}$ at various incubation temperatures. Colourimetric detection was performed after $30 \mathrm{~min}$ incubation with TMB at room temperature and $405 \mathrm{~nm}(n=3)$ rate [40]. It could then be possible that mutated-HRP, due to its lack of complementarity, has more tendency to the nonspecific adsorption on the surface, which enhances its higher and faster hybridisation with the immobilised capture probe at short times and high temperatures through surface diffusion. The concentrations of the target probe would be affected throughout this complex process and therefore could probably tip the balance at some concentrations.

The hybridisation of different concentrations of complementary-HRP and mutated-HRP with biotin-capture probe immobilised on the support was investigated. Above $30 \mu \mathrm{g} \mathrm{mL}^{-1}$ a saturated signal for both targets was obtained. Although the concentration of the target cannot be controlled in a displacement assay (it is precisely what the response is supposed to detect), assuming that the equilibrium is fully reversible, this experiment sets the optimum conditions for the prehybridisation of the suboptimum label: the optimum conditions for prehybridisation chosen were $10 \mu \mathrm{g} \mathrm{mL}{ }^{-1}$ of mutated-HRP in $10 \mathrm{mM}$ Tris- $\mathrm{HCl}$, $1 \mathrm{mM}$ EDTA, $0.3 \times \mathrm{SSC}$ and $2 \times$ Denhardt's solution at $\mathrm{pH} 7.5$ for $1 \mathrm{~h}$ at $50^{\circ} \mathrm{C}$.

\section{Displacement assay}

Once a stable prehybridisation of mutated-HRP was obtained, optimisation of displacement with complementary target oligonucleotide was carried out. To detect displacement two colourimetric detections were performed: first after prehybridisation of mutated-HRP to establish the zero

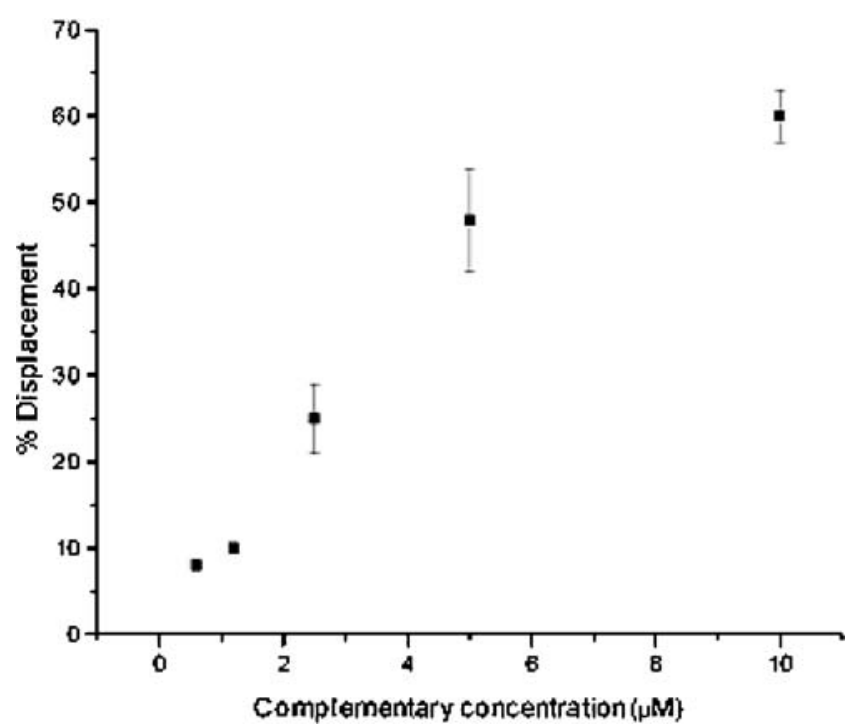

Fig. 6 Absorbance resulting from the displacement of prehybridised mutated-HRP with complementary target or buffer (control). Prehybridisation conditions $10 \mathrm{mg} \mathrm{mL}{ }^{-1}$ mutated-HRP for $1 \mathrm{~h}$ at $50{ }^{\circ} \mathrm{C}$. Displacement conditions $5 \mathrm{~min}$ incubation at $60{ }^{\circ} \mathrm{C}$ at various concentrations of complementary target. Colourimetric detection was performed after $30 \mathrm{~min}$ incubation with $\mathrm{TMB}$ at room temperature at $405 \mathrm{~nm}(n=3)$ 
baselines and second after displacement with complementary target oligonucleotide. Both colourimetric detections were carried out in the same manner i.e. 30 min incubation with TMB detected at $405 \mathrm{~nm}$. The signal obtained after displacement with complementary target or buffer was converted to a percentage of displacement considering the signal from the prehybridised mutated-HRP as $100 \%$ of the signal in each case. Background displacement could occur with buffer solution due to displacement of the equilibrium. Hence, for every measurement a "zero concentration" displacement was established by incubating under the same conditions in the absence of target.

Longer incubation times of complementary target oligonucleotide increased both specific and nonspecific displacement; however, shorter incubation times created greater differences between the nonspecific and specific displacement signal. The highest differences between the specific and nonspecific displacement were found at $5 \mathrm{~min}$ of incubation, at which time the complementary target oligonucleotide displaces $39.3 \%$ of the signal from the prehybridised mutated-HRP and only $7.8 \%$ of signal was displaced by the buffer. (see Fig. 4). Therefore the displacement incubation time was optimised at $5 \mathrm{~min}$ and this value was fixed to examine the effect of temperature on the displacement efficiency.

Figure 5 shows the results obtained for mutated-HRP displacement by complementary target oligonucleotide at different incubation temperatures. As expected, increasing the incubation temperature produced higher destabilisation of prehybridised mutated-HRP; however, nonspecific displacement by the buffer also profited from this destabilisation. After subtracting the value of this "zero" displacement, the highest specific displacement (38.3\%) was achieved at $60{ }^{\circ} \mathrm{C}$. The proportionality between the decrease in signal and the concentration of the complementary target oligonucleotide was verified with the experiments described in Fig. 6.

The above results prove the potential of the displacement detection system as a method to detect label-free oligonucleotide targets with reduced incubation times. Ongoing challenges are the improvement of the system sensitivity and optimisation of the mutated-HRP nonspecific displacement. HRP label used in this displacement assay can be detected colourimetrically and electrochemically, so this approach can be easily adapted to explore electrochemical transduction.

\section{Conclusions}

A novel approach to detect label-free DNA based on suboptimum labelled oligonucleotide displacement was demonstrated. A specific displacement signal of $38.3 \%$ was obtained with the complementary target after thermodynamic and kinetic optimisations of the platform, and the decrease of signal was demonstrated to be proportional to the target concentration. The displacement method achieved the objective of decreasing the response time from $1 \mathrm{~h}$ in direct hybridisation of 19-mer oligonucleotides on direct ELONA to $5 \mathrm{~min}$ in the case of displacement detection.

The application of the displacement methodology shown in this work is a proof of principle of the approach. This platform is still far from being applied to real samples, especially as it has been shown here that each case may require of its own optimisation work. Different length oligonucleotides or different degrees of noncomplementarity require associated efforts to explore their independent hybridisation-dehybridisation conditions. Moreover, real samples that contain a complex matrix, a longer amplified oligonucleotide target and other noncomplementary strands could complicate the specific displacement of the mutated strand; thus this platform should be carefully optimised for a real application. The more simplistic approach to show the potential of the displacement methodology has ongoing challenges such as improving the sensitivity of the system or optimising the mutated-HRP nonspecific displacement. With this aim, stringency conditions of pre-hybridisation of suboptimum labelled oligonucleotide and displacement by the target are being optimized.

Acknowledgements This work has been partially supported by the Commission of the European Communities; project GRD1-2001-4183 "DISSARM".

\section{References}

1. Liu YM, Gong ZL, Morin N et al (2006) Anal Chim Acta 578:7581

2. Barzilai A, Rotman G, Shiloh Y (2002) DNA Repair 1:3-25

3. Jiang B, Li Y, Wu H, He X, Li C, Li L, Tang R, Xie Y, Mao Y (2006) Forensic Sci Int 162:6673

4. Huang RP (2003) Comb Chem High T Scr 6:769-775

5. Brul S, Schuren F, Montijn R (2006) Int J Food Microbiol 112:195199

6. Rodriguez-Mozaz S, de Alba MJL, Barcelo D (2006) Anal Bioanal Chem 386:1025-1041

7. Mao X, Yang L, Su X, Li Y (2006) Biosens Bioelectron 21:11781185

8. Liu SF, Li JR, Jiang L (2005) Colloids Surf A 257:57-62

9. Bianchi N, Rutigliano C, Tomassetti M, Feriotto G, Zorzato F, Gambari R (1997) Clin Diagn Virol 8:199-208

10. Sawata S, Kai E, Ikebukuro K, Iida T, Honda T, Karube I (1997) Nucleic Acids Symp Ser 37:247-248

11. Edwards KA, Baeumner AJ (2006) Anal Bioanal Chem 386:1335-1343

12. Corstjens PLAM, Zuiderwijk M, Nilsson M et al (2003) Anal Biochem 312:191-200

13. Hashimoto K, Ito Y, Ishimori Y (1994) Anal Chem 66:3830-3832

14. Wang J, Jiang M (1998) J Am Chem Soc 120:8281-8282

15. Meric B, Kerman K, Ozkan D, Kara P, Erensoy S, Akarca US, Mascini M, Ozsoz M (2002) Talanta 56:837-846

16. Li H, Rothberg L (2004) Proc Natl Acad Sci USA 101:14036-14039 
17. Yao G, Fang X, Yokota H, Yanagida TW (2003) Chemistry-Eur J 9:5686-5692

18. Yao G, Tan W (2004) Anal Biochem 331:216-223

19. Culha M, Strokes DL, Griffin GD, Dinh TV (2004) Biosens Bioelectron 19:1007-1012

20. Mazid MA, Nishimura K, Nagao K, Jisaka M, Nagaya T, Yokota K (2007) Prostaglandins 83:219-224

21. Liepold P, Wieder H, Hillebrandt H, Hartwich G (2005) Bioelectrochem 67:143-145

22. Hermanson GT (1995) Bioconjugated techniques. Academic, San Diego

23. Aboul-ela F, Koh A, Tinoco I, Martin FH (1985) Nucleic Acids Res 13:4811-4824

24. Gotoh M, Hasegawa Y, Shinohara Y, Shimizu M, Tosu M (1995) DNA Res 2:285-293

25. Peyret N, Sereviratnet PA, Allawi HT, Santa Lucia J Jr (1999) Biochem 38:3468-3477

26. Allawi HT, Santa Lucia J Jr (1998) Nucleic Acids Res 26:26942701

27. Allawi HT, Santa Lucia J Jr (1997) Biochem 36:10581-10594

28. Tawa K, Knoll W (2004) Nucleic Acids Res 32:2372-2377
29. Ke SH, Wartell RM (1993) Nucleic Acids Res 21:5137-5143

30. Peterson AW, Wolf LK, Georgiadis RS (2002) J Am Chem Soc 124:14601-14607

31. Pozhitkov A (2003) Dissertation, University of Cologne

32. Howley PM, Israel MA, Law MF, Martin MA (1979) J Biol Chem 254:4876-4883

33. Breslauer KJ, Frank R, Blocker H, Marky LA (1986) Proc Nat Acad Sci USA 83:3746-3750

34. Maniatis T, Fritsh EF, Sambrook J (1989) Molecular cloning: a laboratory manual. University Press, New York

35. Marky LA, Breslauer KJ (1987) Biopolymers 26:1601-1620

36. Bloomfield VA, Crothers DM, Tinoco I (2000) Nucleic acid structures, properties and functions. University Science Books, Sausalito

37. Dai H, Meyer M, Tepaniants S, Ziman M, Stoughton R (2002) Nucleic Acids Res 30:86-93

38. Maniatis T, Fritsh EF, Sambrook J (1989) Molecular cloning: a laboratory manual. University Press, New York

39. Chan V, Graves DS, McKenzie SE (1995) Biophysical J 69:22452255

40. Bloomfield VA, Crothers DM, Tinoco I (1974) The physical chemistry of nucleic acids. Harper and Row, New York 